\section{Peso ao nascer de recém-nascidos de mães adolescentes comparados com o de puérperas adultas jovens}

\section{Infants' birthweight born from adolescents as compared to those of young adult women}

Samir B. Kassar 1

Ricardo Q. Gurgel 2

Maria de Fátima M. de Albuquerque 3

Marco A. Barbieri 4

Marilia de C. Lima 5

\section{Abstract}

Objectives: to determine maternal age influence on birthweight and other possible associated factors.

Methods: this study is a cohort retrospective study performed in three maternity hospitals in the city of Maceió. The sample consisted of 500 puerperal mothers (250 adolescents and 250 within the age span of 20 to 30 years old) and their newborns.

Results: pregnancy during adolescence was significantly associated to primiparity, to low education level, inadequate prenatal care, low per capita income, and inadequate maternal nutrition. An average birthweight difference of $203 \mathrm{~g}$ plus in newborns of young adult mothers was determined when compared to mothers with ages ranging from 13 to 15 years old, although not significant $(p=0.14)$. Multiple linear regression did not determine significant variation of mother's age in birthweight, nevertheless, variables with significant impact were: maternal nutritional status, prenatal care and infant's gender.

Conclusions: although adolescent mothers have more unfavorable social and economic conditions as compared to young adult mothers, maternal age did not significantly influence birthweight.

Key words Birthweight, Pregnancy in adolescence, Infant, low birth weight, Maternal nutrition, Prematurity

\section{Resumo}

Objetivos: verificar a influência da idade materna no peso ao nascer e de outros possíveis fatores associados.

Métodos: este estudo consiste de uma coorte retrospectiva realizada em três maternidades públicas da cidade de Maceió. A amostra consistiu de 500 puérperas (250 adolescentes e 250 com idade entre 20 a 30 anos) e seus respectivos recém-nascidos.

Resultados: a gravidez na adolescência esteve significantemente associada à primiparidade, à baixa escolaridade, à inadequada assistência ao pré-natal, à baixa renda per capita e ao inadequado estado nutricional materno. Observou-se uma diferença de média do peso ao nascer de $203 \mathrm{~g}$ em favor dos filhos de mães adultas jovens, quando comparados com os das mães com idade entre 13 e 15 anos, embora não significante. Na regressão linear múltipla, a idade materna não influenciou significativamente a variação do peso ao nascer, no entanto, as variáveis que tiveram impacto significativo foram: o estado nutricional materno, a assistência ao pré-natal e o sexo da criança.

Conclusões: apesar das mães adolescentes apresentarem condições socioeconômicas mais desfavoráveis do que as mães adultas jovens, a idade materna não influenciou significativamente no peso ao nascer.

Palavras-chave Peso ao nascer, Gravidez na adolescência, Nutrição materna, Cuidado pré-natal, Prematuro 
Introdução

O peso ao nascer é, isoladamente, um dos fatores mais importantes para morbimortalidade neonatal, com impacto, inclusive, sobre a mortalidade infantil. ${ }^{1}$ Crianças que nascem com peso menor que $2500 \mathrm{~g}$ (baixo peso ao nascer) e com peso situado entre $2500 \mathrm{~g}$ e $2999 \mathrm{~g}$ (peso insuficiente) estão sob alto risco de morrer e adoecer. 1,2

Vários estudos têm mostrado uma forte relação entre a gravidez na adolescência e uma maior freqüência de neonatos com baixo peso ao nascer. Mães com idade inferior a 16 anos aparecem nessas pesquisas com maior risco. ${ }^{3-6}$

Alguns autores consideram que a gravidez na adolescência, por si só, exerce pequeno efeito no peso ao nascer, quando um ou mais fatores de risco são controlados.7,8 Outros, defendem que a gravidez precoce exerce uma influência negativa direta no peso ao nascer. 9,10 Adolescentes muito jovens, eles próprios em crescimento, têm seus recém-nascidos com média de peso inferior em 150-200g, quando comparados com neonatos de mães adultas e adolescentes que estão fora da fase de crescimento.10,11 Parece que adolescentes grávidas menores que 16 anos, em processo de crescimento, competem por nutrientes com seus fetos, e por isso, seus filhos nascem com menor peso, quando comparados com os recém-nascidos de mães adultas e neonatos de adolescentes maiores de 16 anos.9,10

A gravidez na adolescência também tem sido associada à baixa situação socioeconômica, má assistência ao pré-natal, menor ganho de peso na gestação e anemia, quando comparada com as mulheres grávidas fora da adolescência.5,6,12

Devido à associação de fatores de risco, geradores de polêmica fica difícil estabelecer o determinante - se exclusivamente biológico e/ou social - nos piores resultados perinatais das grávidas adolescentes.

Investigações sobre os mecanismos explicativos da associação entre o menor peso ao nascer e a gravidez na adolescência devem ser realizadas, abrangendo fatores sócio-culturais, como pobreza e marginalidade social, assim como os de natureza biológica, na gestação. ${ }^{13}$

Neste estudo pretende-se comparar a média do peso ao nascer entre os neonatos de mães adolescentes com a dos recém-nascidos das adultas jovens e verificar dentre os fatores sócio-demográficos e obstétricos, assim como o hábito de fumar e/ou beber na gravidez, aqueles que possam determinar a variação do peso ao nascer.

\section{Métodos}

Estudo de coorte retrospectivo foi o desenho utilizado para comparar a média do peso ao nascer de neonatos de mães adolescentes ( $<20$ anos) com a dos recém-nascidos de mães adultas jovens ( 20 a 30 anos). Este grupo de comparação foi escolhido por corresponder à melhor época para a procriação do ponto de vista social e biológico.

O trabalho foi realizado na cidade de Maceió, capital do Estado de Alagoas, Nordeste do Brasil, em três maternidades que atendem gestantes do Sistema Único de Saúde (SUS). Foram excluídas as gestações gemelares e aquelas com suspeita de infecções congênitas ou que resultaram em recémnascidos malformados. Puérperas com informações incompletas também foram excluídas do estudo.

O critério de seleção adotado foi o de natureza aleatória, utilizando-se para cada adolescente puérpera identificada na enfermaria no dia da entrevista o registro seguinte de outra mãe com idade entre 20 a 30 anos.

Foram estudadas 500 mães (250 para cada grupo) que se encontravam no puerpério com seus respectivos recém-nascidos, no período de julho a outubro de 1996. Em algumas análises se comparou o grupo adulto jovem com dois grupos de adolescentes: um de 13 a 15 anos e o outro de 16 a 19 anos.

O tamanho mínimo da amostra foi de 215 mães para cada grupo, conforme Kirkwood, 14 utilizandose um poder de $80 \%$ e nível de significância de $5 \%$ para detectar uma diferença de médicas estatisticamente significante entre os grupos. Definiu-se que a diferença de média de peso ao nascer esperada entre os dois grupos estudados seria de $100 \mathrm{~g}$, assumindose um desvio padrão do peso ao nascer de recémnascidos de mães adultas jovens de $420 \mathrm{~g}$ e de $469 \mathrm{~g}$ para o desvio padrão do peso de neonatos de mães adolescentes. ${ }^{8}$

O peso foi avaliado pelo pediatra logo após o nascimento, em balança, com sensibilidade de $10 \mathrm{~g}$. As balanças foram aferidas semanalmente com um peso de $2 \mathrm{~kg}$ cedido pelo INMETRO. Enfermeiras dos hospitais onde se realizou a pesquisa foram orientadas a regular as balanças diariamente.

As mães foram entrevistadas, após o parto, e preencheram formulários contendo questões fechadas e pré-codificadas. O uso do álcool e do fumo foi estudado mediante sua ocorrência sem levar em consideração a quantidade consumida e o tempo de exposição durante a gravidez. A escolaridade materna e a renda per capita foram os fatores sócio-demográficos pesquisados. A expressão "salário" refere-se ao salário mínimo vigente no 
País. Foram consideradas com baixa escolaridade as mães analfabetas e as com menos de quatro anos de escolaridaede. Considerou-se pré-natal insatisfatório quando a gestante freqüentou menos que seis consultas. Mães com perímetro braquial menor ou igual a $25 \mathrm{~cm}$ foram consideradas como pior estado nutricional, segundo recomendação de Batista Filho et al. ${ }^{15} \mathrm{~A}$ idade gestacional foi determinada pela data da última menstruação (DUM) e confirmada com exame físico do recém-nascido através do método de Capurro. A idade ginecológica foi a idade cronológica menos a idade da menarca.

Os dados foram analisados por intermédio dos programas no Epi-info 6.0 e o SPSS versão 8,0. O intervalo de confiança de $95 \%$ foi utilizado para variáveis quantitativas. O teste de significância usado para comparar diferença de proporções foi o qui-quadrado. Na comparação de médias entre dois grupos utilizou-se o teste $\mathrm{t}$ de Student e a análise de variância na comparação de médias entre mais de dois grupos. A análise de regressão linear múltipla foi utilizada para estudar a associação entre a idade materna e o peso ao nascer, controlando-se o efeito das demais variáveis maternas envolvidas no processo. Para tanto, o peso ao nascer foi considerado como variável dependente. As variáveis maternas que apresentaram associação com o peso ao nascer nas análises bivariadas com um nível de significância $<0,20$ foram incluídas nos modelos de regressão. A entrada das variáveis independentes foi realizada considerando-se três modelos: no modelo um, o impacto da renda per capita no peso ao nascer foi observado isoladamente; no modelo dois, a renda per capita foi considerada conjuntamente com as outras variáveis maternas (idade cronológica e idade ginecológica materna, circunferência de braço, paridade, número de consultas no pré-natal); e, no modelo três, foram incluídas todas as variáveis anteriores mais o sexo das crianças. O nível de significância adotado foi de $p<0,05$.

O estudo obteve a aprovação da Comissão de Ética da Universidade Federal de Alagoas.

\section{Resultados}

A faixa etária do grupo de mães adolescentes variou de 13 a 19 anos. A idade predominante foi 18 anos $(23,6 \%)$. O grupo menor que 16 anos representou $18,4 \%$ da amostra. A idade de mães adultas jovens variou de 20 a 30 anos e a idade predominante foi de 24 anos $(15,2 \%)$.

O hábito de fumar e o parto cesáreo foram mais freqüentes nas mães adultas jovens $(p<0,05)$. O uso do álcool na gravidez, a prematuridade $(<37$ semanas) e o baixo peso ao nascer $(<2500 \mathrm{~g})$ não apresentaram diferença estatística entre os grupos de mães. A primiparidade, a baixa escolaridade, o prénatal insatisfatório ( $<6$ consultas), a baixa renda per capita e o pior estado nutricional apresentaram as-

Fatores demográficos, hábitos de saúde e obstétricos da amostra. Maceió, Alagoas, 1996.

\begin{tabular}{|c|c|c|c|c|c|}
\hline & \multicolumn{2}{|c|}{ Adolescente } & \multicolumn{3}{|c|}{ Adulta } \\
\hline & \multicolumn{2}{|c|}{$N=250$} & \multicolumn{3}{|c|}{$N=250$} \\
\hline & $\mathrm{n}$ & $\%$ & $\mathrm{n}$ & $\%$ & $p^{*}$ \\
\hline Álcool na gravidez & 17 & 6,8 & 25 & 10,0 & 0,19 \\
\hline Mães analfabetas e $<4$ anos de escola & 227 & 90,8 & 179 & 71,6 & $<0,001$ \\
\hline Renda per capita que $1 / 2$ salário mínimo & 134 & 53,6 & 111 & 44,4 & 0,039 \\
\hline Fumo na gravidez & 20 & 8,0 & 44 & 17,6 & 0,001 \\
\hline Pré-natal insatisfatório (<6 consultas) & 137 & 54,8 & 113 & 45,2 & 0,031 \\
\hline Circunferência do braço $25 \mathrm{~cm}$ & 131 & 36,0 & 84 & 21,6 & $<0,001$ \\
\hline Primiparidade & 167 & 66,8 & 68 & 27,2 & $<0,001$ \\
\hline Parto cesáreo & 78 & 31,2 & 107 & 42,8 & 0,007 \\
\hline Prematuridade (<37 semanas) & 29 & 11,6 & 32 & 12,8 & 0,68 \\
\hline Baixo peso ao nascer $(<2500 \mathrm{~g})$ & 39 & 15,6 & 36 & 14,4 & 0,80 \\
\hline
\end{tabular}

*Qui-quadrado 
Tabela 2

Médias de peso ao nascer de recém-nascidos de acordo com as variáveis socioeconômicas, reprodutiva, estado nutricional materno e sexo das crianças. Maceió, Alagoas, 1996.

\begin{tabular}{|c|c|c|c|c|c|c|}
\hline & $\mathrm{N}$ & $\bar{x}$ & \pm & $d p$ & $\mathrm{IC} 95 \%$ & Teste de significância \\
\hline \multicolumn{7}{|c|}{ Idade materna (anos) } \\
\hline 13 a 15 & 46 & 2877 & \pm & 629 & $2691-3062$ & $F=1,96$ \\
\hline 16 a 19 & 204 & 3033 & \pm & 619 & $2947-3119$ & $p=0,14$ \\
\hline 20 a 30 & 250 & 3080 & \pm & 666 & $2997-3162$ & \\
\hline \multicolumn{7}{|l|}{ Primiparidade } \\
\hline Sim & 235 & 2991 & \pm & 630 & $2910-3072$ & $t=-1,66$ \\
\hline Não & 265 & 3087 & \pm & 656 & $3008-3166$ & $p=0,09$ \\
\hline \multicolumn{7}{|c|}{ Idade ginecológica (anos) } \\
\hline 2 & 36 & 2856 & \pm & 666 & $2631-3082$ & $t=-1,78$ \\
\hline$>2$ & 428 & 3055 & \pm & 644 & $2994-3116$ & $p=0,07$ \\
\hline \multicolumn{7}{|c|}{ Consultas no pré-natal } \\
\hline$<6$ & 250 & 2957 & \pm & 639 & $2878-3037$ & $t=-2,96$ \\
\hline 6 & 250 & 3127 & \pm & 641 & $3047-3207$ & $p=0,003$ \\
\hline \multicolumn{7}{|c|}{ Circunferência do braço $(\mathrm{cm})$} \\
\hline 25 & 144 & 2899 & \pm & 653 & $2791-3006$ & $t=-3,19$ \\
\hline$>25$ & 356 & 3100 & \pm & 633 & $3034-3166$ & $p=0,002$ \\
\hline \multicolumn{7}{|c|}{ Renda per capita mensal (SM) } \\
\hline $1 / 4$ & 70 & 2957 & \pm & 661 & $2800-3115$ & $F=2,3$ \\
\hline$>1 / 4$ a $1 / 2$ & 175 & 2988 & \pm & 610 & $2897-3079$ & $P=0,09$ \\
\hline$>1 / 2$ & 255 & 3102 & \pm & 660 & $3021-3184$ & \\
\hline \multicolumn{7}{|l|}{ Sexo } \\
\hline Masculino & 266 & 3118 & \pm & 633 & $3041-3195$ & $t=2,7$ \\
\hline Feminino & 233 & 2964 & \pm & 638 & $2882-3046$ & $P=0,007$ \\
\hline
\end{tabular}

$\mathrm{SM}$ = salários mínimos; $\mathrm{F}=$ Análise de variância; $\mathrm{t}$ = Teste " $\mathrm{t}$ " de Student

sociação estatisticamente significante com a gravidez na adolescência $(p<0,05)$ (Tabela 1$)$.

A média do peso ao nascer de filhos das mães adolescentes foi de 3004g (DP 623) contra 3080g (DP 666) para os das mães adultas jovens. A pequena diferença de $76 \mathrm{~g}$ a favor dos recém-nascidos de mães mais velhas não foi significante.

A média do peso ao nascer tendeu a aumentar com o aumento da idade materna, verificando-se uma diferença de $203 \mathrm{~g}$ a menos entre os filhos de mães na faixa etária entre 13 a 15 anos, quando comparados com os das mães entre 20 a 30 anos de idade, também não significante. Recém-nascidos cujas mães fizeram menos de seis consultas no prénatal, foram primíparas, tiveram o perímetro braquial menor ou igual a $25 \mathrm{~cm}$, ganharam menos que meio salário per capita, tinham idade ginecológica menor ou igual a dois anos e pariram recém-nascidos do sexo feminino, tiveram menores médias de peso (Tabela 2).

Na Tabela 3, observa-se que o percentual de baixo peso ao nascer foi mais elevado no grupo das adolescentes mais jovens ( $p=0,03)$.

Os resultados da regressão linear múltipla podem 
Distribuição da proporção de recém-nascidos com baixo peso ao nascer de mães adolescentes e mães adultas jovens. Maceió, 1996.

\begin{tabular}{|c|c|c|c|c|c|}
\hline \multirow[b]{3}{*}{ Idade materna } & \multicolumn{5}{|c|}{ Peso ao nascer } \\
\hline & \multicolumn{2}{|c|}{$<2500 \mathrm{~g}$} & \multicolumn{2}{|c|}{$\geq 2500 \mathrm{~g}$} & \multirow[t]{2}{*}{ Total } \\
\hline & $\mathrm{n}$ & $\%$ & $\mathrm{n}$ & $\%$ & \\
\hline $13-15$ anos & 13 & 28,2 & 33 & 71,8 & 46 \\
\hline $16-19$ anos & 26 & 12,8 & 178 & 87,2 & 204 \\
\hline $20-30$ anos & 36 & 14,4 & 214 & 85,6 & 250 \\
\hline Total & 75 & 15,0 & 425 & 85,0 & 500 \\
\hline
\end{tabular}

$\chi^{2}=7,23 ; p=0,03$

ser vistos na Tabela 4 . O modelo 3 mostra que a idade materna não afetou significativamente a variação do peso ao nascer após ajuste pelas demais variáveis. As variáveis que tiveram um impacto significativo na variação do peso ao nascer foram o estado nutricional materno, a assistência ao pré-natal e o sexo. Essas variáveis conjuntamente explicariam $7,1 \%$ da variação do peso ao nascer.

Tabela 4

Regressão linear múltipla do peso ao nascer com as variáveis socioeconômicas, demográficas, reprodutiva e nutricional materna e sexo da criança.

Variáveis independentes

Coeficientes de regressão

\begin{tabular}{lccc}
\hline & Modelo 1 & Modelo 2 & Modelo 3 \\
\hline Renda per capita ( 0,5 salário mínimo) & $-117,0^{*}$ & $-28,5$ & $-34,5$ \\
Idade ginecológica ( 2 anos) & & $-194,0$ & $-189,0$ \\
Idade materna (<16 anos) & 83,1 & 79,8 \\
Circunferência do braço ( 25cm) & $-253,0+$ & $-241,0+$ \\
Paridade (primiparidade) & $-72,0$ & $-70,0$ \\
Pré-natal (<6 consultas) & & $-122,6^{*}$ & $-124,6^{*}$ \\
Sexo (feminino) & $-119,2^{*}$ & & \\
\hline
\end{tabular}

Coeficientes de determinação (Mod 1, R2=0,8\%; $\operatorname{Mod} 2, R^{2}=6,3 \% ; \operatorname{Mod} 3, R^{2}=7,1 \%$ ); ${ }^{+} p<0,001 ;{ }^{*} p<0,05$.

\section{Discussão}

A média de peso dos recém-nascidos filhos de mães adolescentes foi menor em 76g, quando comparada com a média dos pesos dos neonatos de mães adultas. Entre as mães mais jovens, que poderiam elas próprias estar em crescimento, a diferença de média foi maior (203g), quando comparada com a média de peso dos bebês das mães adultas. Outros estudiosos fizeram esta mesma comparação e encontraram dife- renças de 150-200g e 310g. Nesses estudos, os recémnascidos de mães adolescentes sempre tiveram média de peso inferior quando comparados com os filhos de mães adultas. No presente estudo, no grupo das adolescentes menores que 16 anos, sobre o qual os efeitos biológicos e sociais poderiam estar exercendo maiores influências no peso ao nascer, encontrou-se uma média de peso um pouco mais inferior àquela dos recém-nascidos de mães adultas, porém sem significância estatística. 
A freqüência de baixo peso ao nascer foi maior no grupo de adolescentes mais jovens (13 a 15 anos). Já no grupo de adolescentes de 16 a 19 anos o baixo peso ao nascer teve uma freqüência de $12,8 \%$, e entre as adultas jovens, $14,4 \%$. A literatura concorda que, biologicamente, as adolescentes com mais de 17 anos têm um comportamento reprodutivo semelhante ao das adultas. O nosso achado de maior frequiência de recém-nascidos com baixo peso entre as adolescentes mais jovens (16 anos) é semelhante ao de vários outros estudos. 13,19

Esses resultados indicam que o grupo de grávidas adolescentes ( $<20$ anos) não é homogêneo. Existe um conjunto de mães (menores de 16 anos) que aparentemente é mais exposto a riscos, e tal questão merece ser estudada separadamente como recomendam Phipps e Sower. 6 Esses autores sugerem a necessidade de um maior esclarecimento sobre quais fatores, se biológico e/ou social, expõem mães adolescentes muito jovens a maiores riscos de ter filhos com baixo peso.

É contraditória a influência da idade materna no baixo peso ao nascer. $\mathrm{O}$ grupo de mães adolescentes tem sido considerado de maior risco para o baixo peso ao nascer, mesmo quando controlado por outras variáveis 13,17,19. No entanto, outros estudos sugerem que a gestação na adolescência não é uma causa direta ou determinante independente no baixo peso ao nascer. $7,8,16$

A idade cronológica, por si só, não é um bom fator preditivo na determinação do peso ao nascer, devendo ser considerada juntamente com fatores outros, mais especificamente os socioeconômicos. Estes estiveram, nas análises realizadas, significativamente associados às menores médias de peso ao nascer. Todos esses fatores podem interferir no crescimento fetal. Nesse sentido, os resultados da regressão múltipla mostraram que, apesar do impacto das variáveis maternas sobre o valor do peso ao nascer da criança estar manifesto, de uma forma geral a análise desse impacto é uma questão complexa, especialmente quando muitas variáveis maternas são consideradas simultaneamente.

A complexidade é decorrente de se estar avaliando a expressão biológica de um evento que não acontece isoladamente de outras dimensões. A análise de eventos biológicos, especialmente os relacionados ao desenvolvimento humano e à gravidez na adolescência, deve seguir um modelo que contemple as várias situações existentes na vida de um indivíduo. É fundamental considerar a interação das várias dimensões, tais como a socioeconômica, psicológica e cultural sobre as respostas biológicas consideradas e/ou estudadas. A grande diversidade de situações encontradas dentro de um grupo social pode interferir, e conferir, ao mesmo tempo, diferentes respostas nas manifestações biológicas desse grupo.12,13

Neste estudo, a gravidez na adolescência em menores de 16 anos, por si só, não foi suficiente para explicar o baixo peso ao nascer das crianças, já que outros fatores não foram controlados. A diferença de média de peso não estatisticamente significante deveu-se possivelmente a um conjunto relativamente pequeno de mães menores de 16 anos contempladas na pesquisa, diferentemente do que se encontrou quando se fez uma categorização dos pesos. A idade materna não influenciou significativamente no peso ao nascer. Os fatores socioeconômicos, tais como o pior estado nutricional e o pré-natal insatisfatório, que tiveram associação com a gravidez na adolescência, foram as variáveis que mostraram impacto negativo na determinação do peso ao nascer. O maior benefício para esse grupo se-ria a melhoria de suas condições socioeconômicas e maior oferta de serviço de atenção ao pré-natal.

Estudos específicos para comparar o produto da gestação de adolescentes grávidas mais jovens $(<16$ anos) com o das mais velhas, levando em consideração as diferenças socioeconômicas, são imprescindíveis para averiguar o real papel biológico da adolescência no crescimento fetal.

\section{Referências}

1. WHO (World Health Organizacion). The incidence of low birth weight: a critical review of available information. World Health Stat Ann 1980; 33: 197-244.

2. Puffer RR, Serrano, CV. El peso al nascer, la edad materna y el orden de nascimento: três importantes factores de la mortalidad infantil. Washington (DC); 1975. (Publicación Científica, n. 294).

3. Fraser AM, Brockert JE, Ward RH. Association of young maternal age with adverse reproductive autcomes. N Engl J Med 1995; 332: 1113-7.

4. Ress JM, Lederman SA, Kiely JL. Peso de nascimento associado à taxa de mortalidade mais baixa: filhos de mães adolescentes e adultas. Pediatrics 1997; 1: 261-8. [edição portuguesa].

5. Ribeiro ERRO, Barbieri MA, Bettiol H, Silva AAM. Comparação entre duas coortes de mães adolescentes em município do sudoeste do Brasil. Rev Saúde Pública. 2000; 34: $136-42$.

6. Phipps MG, Sowers M. Defining early adolescent childbearing. Am J Public Health 2002; 92: 125-8. 
7. Kramer MS. Determinants of low birth weight methodological assessment and meta analyses. Bull World Health Organ 1987; 65: 663-737.

8. Lima MC. Influence of maternal work activity on birth weight in Palmares - Northeast - Brazil [Thesis PhD]. London: London School of Higiene and Tropical Medicine; 1995.

9. Frisancho AR, Matos J, Bolletinao L A. Influence of growth status and placental function on birth weight of infants Born to still growing teenagers. Am J Clin Nutr 1984; 40: 801-7.

10. Scholl TO, Hediger ML, Schall JI. Mechanisms for maternal age differences in birth weight. Am J Epidemiol1996; 143: 842-3.

11. Scholl TO, Hediger ML, Schall JI, Khoo CS, Fischer RL Maternal growth during pregnancy and the competition for nutrients. Am J Clin Nutr 1994; 60: 183-8.

12. Gama SGN, Szwarcwald CL, Leal MC. Experiência de gravidez na adolescência, fatores associados e resultados perinatais entre puérperas de baixa renda. Cad Saúde Pública 2002; 18: 153-61

13. Gama SGN, Szwarcwald CL, Leal MC, Theme Filha, MM. Gravidez na adolescência como fator de risco para baixo peso ao nascer no município do Rio de Janeiro, 1996 a 1998. Rev Saúde Pública 2001; 35: 74-80.

Recebido em 10 de novembro de 2004

Versão final apresentada em 5 de julho de 2005

Aprovado em 15 de agosto de 2005
14. Kirkwood BR. Essential of medical statistics. Oxford: Blackwell Scientific; 1988.

15. Batista Filho M, Barros LF, Nácul LC. O perímetro braquial como método de avaliação do estado nutricional em gestantes. Rev IMIP 1993; 7: 12-6.

16. Mariotoni GGB, Barros Filho AA. A gravidez na adolescência é fator de risco para o baixo peso ao nascer? (Brasil). Rev Chil Pediatr 2000, 5: 453-60.

17. Solla JJSP, Pereira RAG, Medina MG, Pinto LLS. Análisis multifactorial de los factores de riesgo de bajopeso al nacer en Salvador, Bahia. Rev Panam Salud Publica 1997, 2: 1-6.

18. Buschaman NA, Foster G, Vickers P. Adolescent girls and their babies: achieving optimal birthweight. Gestacional weight gain and pregnancy outcome in terms of gestation at delivery and infant birth weight: a comparisosn between adolescents under 16 and adult women. Child Care Health Dev 2001; 27: 163-71.

19. Costa MCO, Santos CAT, Sobrinho CLN, Freitas JO, Ferreira KASL, Silva MA, Paula PLB. Estudos dos partos e nascidos vivos de mães adolescentes e adultas jovens no município de Feira de Santana, Bahia, Brasil. Cad Saúde Pública 2002; 18: 715-22. 